\title{
Errata
}

\section{Modeling dependence in finance and insurance: the copula approach}

\author{
Dietmar Pfeifer and Johana Nešlehová (Oldenburg)
}

Blätter der DGVFM Band XXVI, Heft 2 (2003), 177 - 191.

1. Theorem 4.1 should read correctly:

Theorem 4.1. Let $\varphi$ be a continuous strictly decreasing function on the interval $(0,1]$ such that $\varphi(1)=0$ and $\lim _{z \downarrow 0} \varphi(z)=\infty$, and let $\varphi^{-1}$ denote its inverse on the interval $[0, \infty)$. If $C$ is a function on the unit $n$-cube $[0,1]^{n}$ with $n \geq 3$, fulfilling the above additive relationship, then $C$ is a copula if and only if $\varphi^{-1}$ is completely monotonic, i.e.

$$
(-1)^{k} \frac{d^{k}}{d s^{k}} \varphi^{-1}(s) \geq 0 \text { for all } k \in \mathbb{N} \text { and } s>0 .
$$

In this case, we have necessarily $C \succ \Pi$ (in the sense of Definition 3.2).

In fact, for dimension $n=2$, the situation is a little simpler here since just convexity of $\varphi$ is sufficient for $C$ being a copula. Also, $C \prec \Pi$ is possible here. For details, see Theorem 4.1.4 in Nelsen [1].

2. The following misprints should be corrected as follows:

The Clayton copula on p. 186 , line 8 should read:

$$
C\left(u_{1}, \cdots, u_{n}\right)=\left[\sum_{i=1}^{n} u_{i}^{-\theta}-n+1\right]^{-1 / \theta}, \mathbf{u} \in(0,1]^{n}, \theta>0
$$

(likewise for the table on the same page, line 19).

The Gumbel copula on p. 186 , line 20 should read:

$$
\exp \left(-\left\{\sum_{i=1}^{n}\left(-\ln \left(u_{i}\right)\right)^{\theta}\right\}^{1 / \theta}\right), \theta \geq 1
$$

Reference

[1] Nelsen, R.B.: An Introduction to Copulas. Lecture Notes in Statistics 139, Springer, N.Y. (1999). 